\title{
Intrications organo-psychiatriques : le concept de Troubles Psychiatriques Complexes, quels examens complémentaires ? (Partie 1)
}

\author{
FERRERI F(1 $)^{1}$, BOURLA A $(1)^{1}$, CAPRON J², QUILLEROU B ${ }^{1}$, ROSSIGNOL J ${ }^{3}$, BORDEN A ${ }^{2}$, GUECHOT J ${ }^{4}$,
}

LAMAZIERE A ${ }^{4}$, NUSS P $\mathrm{P}^{1,6,7}$, MEKINIAN A ${ }^{5}$, MOUCHABAC $\mathrm{S}^{1}$

\author{
1. Sorbonne Université, Paris Univ-06, Paris, Service de Psychiatrie et de Psychologie médicale, Hôpital Saint-Antoine, \\ 75012, Paris, France \\ 2. Sorbonne Université, Paris Univ-06, Paris, Service de Neurologie, Hôpital Saint-Antoine, 75012, Paris, France \\ 3. Service hématologie, Hôpital Necker Enfant Malades, Université Paris 5, Paris, France. \\ 4. Sorbonne Université, Paris Univ-06, Paris, Service de Biochimie, Hôpital Saint-Antoine, 75012, Paris, France \\ 5. Sorbonne Université, Paris Univ-06, Paris, Service de Médecine interne, Hôpital Saint-Antoine, 75012, Paris, France \\ 6. INSERM ERL 1157, CHU Saint-Antoine, Paris, France \\ 7. UMR 7203, Laboratoire des biomolécules, Sorbonne Universités-UPMC Université Paris 06, Paris, France
}

(1) Les auteurs ont contribué à part égale

Résumé : Si des manifestations psychiatriques en lien avec des pathologies organiques sont bien connues (syndrome maniaque et hyperthyroïdie, syndrome dépressif et insuffisance corticotrope, trouble anxieux et maladie cardiaque, etc.) d'autres maladies psychiatriques peuvent être secondaires à des pathologies neurologiques, auto-immunes, métaboliques, paranéoplasiques ou endocriniennes. Les manifestations précoces peuvent alors être essentiellement psycho-comportementales avant d'être neurologiques ou systémiques. De plus, un grand nombre de facteurs (nutritionnels, toxiques, immunologiques, etc.) qui exercent une influence sur les liens existants entre organicité et pathologies psychiatriques ne sont pas systématiquement recherchés. Les objectifs de cet article sont de décrire des troubles psychiatriques complexes, de rappeler les explorations «classiques minimales» en psychiatrie, de proposer un rationnel sur le concept de «troubles psychiatriques complexes » et de proposer une méthode d'exploration systématisée. Cet article a été élaboré avec des internistes, des neurologues, des hématologues, des psychiatres et des biologistes afin d'optimiser la prise en charge médicale de ces patients chez qui le diagnostic psychiatrique masque une cause organo-psychiatrique curable.

Abstract: If psychiatric manifestations related to organic pathologies are well known (manic syndrome and hyperthyroidism, depressive syndrome and corticotropic insufficiency, anxiety disorder and heart disease, etc.) other psychiatric disorders may be secondary to neurological, autoimmune, metabolic, paraneoplastic or endocrine diseases with early psycho-behavioral manifestations before neurological or systemic manifestations. Furthermore a large number of factors (nutritional, toxic, immunological, etc.) contribute to the mutual influence between organicity and psychiatric pathologies. After exposing situations involving complex psychiatric disorders and recalling "classical" explorations in psychiatry, the aim of this article is to propose a rational for creating a common object of clinical reflection ("complex psychiatric disorders") and an exploration method developed with internists, neurologists, hematologists, psychiatrists and biologists to optimize the medical management of these patients in whom the psychiatric diagnosis eclipses a curable organo-psychiatric cause.

Nombre de caractère : 38343

Conflit d'intérêt : Les auteurs déclarent n'avoir aucun conflit d'intérêt 


\section{Manuscrit}

\section{Introduction}

Si dans la plupart des cas les troubles psychiatriques sont considérés comme idiopathiques et plurifactoriels, de nombreuses études suggèrent qu'il existe des niveaux d'organisation multiples (biologiques, psychologiques, sociaux, etc.) [1] et il est reconnu que certaines maladies psychiatriques peuvent être secondaires à des pathologies neurologiques, auto-immunes, métaboliques, paranéoplasiques ou endocriniennes [2,3]. Dans ces situations, les manifestations précoces de la maladie sont essentiellement psycho-comportementales avant d'être neurologiques ou systémiques. Parmi ces pathologies que l'on pourrait qualifier «d'organo-psychiatriques», certaines ont un mauvais pronostic à court terme avec une morbi-mortalité élevée et de nombreuses situations nécessitent des décisions thérapeutiques urgentes avec un traitement spécifique, ainsi qu'une approche pluridisciplinaire favorisant la coopération entre différentes spécialités médicales et la psychiatrie. Historiquement, la psychiatrie ayant été marquée par une opposition entre les partisans d'une approche psychologique pure et les tenants d'une approche organique (pour lesquels l'étiologie des troubles psychiatriques trouve son origine dans des dysfonctionnements biologiques cérébraux), cette dichotomie a retardé l'élaboration d'une approche plus intégrative. L'essor récent des neurosciences a enrichi les modèles d'interactions causales en montrant qu'organicité et pathologies psychiatriques s'influencent mutuellement et sont le résultat d'interactions entre un individu, sa vulnérabilité et un environnement qui recouvre de nombreux facteurs (nutritionnels, toxiques, immunologiques, etc.). Si des manifestations psychiatriques en lien avec des pathologies organiques sont bien connues (syndrome maniaque secondaire à une hyperthyroïdie, syndrome dépressif en lien avec une hypothyroïdie, une insuffisance corticotrope ou un cancer, pathologie cardiaque ou pulmonaire se manifestant comme un trouble anxieux, etc.) d'autres le sont moins mais méritent qu'on s'y intéresse tant elles impactent négativement le pronostic de certains patients chez qui le diagnostic psychiatrique éclipse une cause organo-psychiatrique curable. 
L'objectif principal de cet article est de proposer, après avoir exposé les situations impliquant des troubles psychiatriques complexes, un rationnel permettant de créer un objet commun de réflexion clinique (les «troubles psychiatriques complexes ») et une méthode d'exploration élaborés avec des internistes, des neurologues, des hématologues, des psychiatres et des biologistes afin d'optimiser la prise en charge médicale de ces patients.

\section{Les intrications organo-psychiatriques}

Plusieurs études retrouvent qu'environ $10 \%$ des patients admis en unité d'hospitalisation psychiatrique présentent en réalité des troubles organiques à l'origine des manifestations psychiatriques $[4,5] . \mathrm{Si}$ dans un grand nombre de cas il s'agit de causes en lien avec des toxiques (ou des syndromes de sevrage), il n'est pas rare que d'autres types de troubles en soit à l'origine, notamment des maladies neurologiques (épilepsie, encéphalites), infectieuses (neuroméningées, digestive ou pulmonaire) ou endocrino-métabolique (dysthyroïdie, dysnatrémie). Si la plupart des maladies décrites dans ces étude sont pourtant bien connue comme pourvoyeuses de symptômes psychiatriques, et que le défaut de reconnaissance de la pathologie à l'origine des signes observés est à mettre sur le compte d'erreurs d'appréciation de la situation clinique (défaut d'examen physique dans $40 \%$ des cas, absence d'examen complémentaire approprié dans plus de 30\% des cas) [6], dans d'autres situations les intrications sont plus complexes à mettre en évidence. Notre expérience nous montre que pour certains patients il peut se passer jusqu'à 10 ans avant que le diagnostic soit redressé, notamment en cas de trouble neuro-métabolique ou auto-immun. Pour illustrer ces difficultés, nous pouvons ainsi rapporter le cas d'une jeune patiente de 16 ans qui avait été admise pour une symptomatologie dépressive et des mouvements anormaux du visage qui avaient été étiquetés d'abord comme des tics puis comme des manifestations «d'hystérie » sans aucun autre symptôme neurologique, avec un bilan sanguin, une IRM, un EEG et une PL (glycorrachie, protéinorrachie, culture) sans particularité, résistante aux traitements médicamenteux mais avec une certaine amélioration après un traitement par ECT, et qui s'était en réalité avéré être une encéphalite à anticorps anti-NMDAr paranéoplasique d'un tératome de l'ovaire. On pourrait aussi rapporter le cas de ce patient de 28 ans hospitalisé depuis 6 ans sur un secteur psychiatrique pour une schizophrénie déficitaire et qui présentait en réalité une maladie de 
Niemann Pick de type C. Des cas comme ceux-là ne sont pas anecdotiques, et ils touchent un nombre suffisant de patients pour que se développent des programmes et des procédures standardisées d'exploration et de prise en charge de ces intrications.

\section{Le bilan de $1^{\text {ère }}$ intention}

Le bilan proposé par la Haute Autorité de Santé HAS (tableau 1) au titre du bilan initial d'un trouble dépressif sévère, d'un trouble anxieux sévère, d'un trouble bipolaire ou d'un trouble du spectre de la schizophrénie, permet d'éliminer certaines causes organiques fréquentes et de rechercher des comorbidités influençant le choix des traitements (bilan pré-thérapeutique) [7]. La fédération française de psychiatrie aussi a proposé des recommandations de bonne pratique essentiellement à destination des psychiatres car s'intéressant à la prise en charge des patients ayant une pathologie psychiatrique sévère et chronique, mais il nous semble approprié de les citer ici tant l'incidence des troubles qu'elle se propose de dépister est importante chez les patients atteints de troubles psychiatriques et présente donc un intérêt de santé public en plus d'impacter fortement le pronostic du patient [8]. Ils insistent particulièrement sur le suivi cardiométabolique des patients traités par psychotropes et reprennent de facto les propositions émises par l'HAS à ce sujet (bilan lipidique, glycémie à jeun, tension artérielle, périmètre ombilical, poids, IMC, etc.). Enfin notre groupe de travail propose de compléter ce bilan minimal par une précision sur la place de l'imagerie cérébrale et par la recherche systématique de syphilis vu l'évolution épidémiologique actuelle et a fortiori en cas de premier épisode.

\section{Tableau 1. Bilan minimal de $1^{\text {ère }}$ intention}

\section{Examen Rationnel}

\section{Recommandé par la HAS}

NFS

Ionogramme, $\mathbf{C a}^{2+} \quad$ Symptomatologie neurovégétative, anxiété : dys-natrémie, -kaliémie, -calcémie

Créatininémie Avec DFG (CKD-EPI) pour évaluer une atteinte rénale (pré-thérapeutique)

TSH

Dépression, irritabilité, euphorie, trouble pondéral : dysthyroïdie

$\mathbf{T 3}-\mathbf{T 4}^{\mathbf{a}}$

Bilan systématique d'un trouble dépressif bipolaire 


\footnotetext{
Albumine (et pré-) Episode dépressif avec anorexie, dénutrition (bilan systématique)

Bilan hépatique Pour évaluer une atteinte hépatique (pré-thérapeutique)

Bilan lipidique Pré-thérapeutique et recherche de complication du traitement

Glycémie à jeun Pré-thérapeutique, et recherche de complication du traitement

BHCG Chez toute femme en âge de procréer au titre du bilan pré-thérapeutique

IRM cérébrale Indiquée pour tout premier épisode psychiatrique

Polysomnographie Exploration des troubles du sommeil associés à la dépression chronique, cause de la dépression ou secondaires

ECG Bilan initial et suivi des effets indésirable des traitements

\section{Recommandé par la Fédération Française de Psychiatrie}

CRP Recherche d'inflammation

VIH, VHC, VHB Recherche d'infection

Toxiques urinaires Recherche d'intoxication

Prolactine $^{\mathbf{b}} \quad$ Recherche de complication du traitement par antipsychotique / neuroleptique

\section{Recommandé par notre groupe de travail}

EEG Proposé pour tout premier épisode psychiatrique sévère

Sérologie Recherche d'infection syphilitique

tréponémique

a. Uniquement chez les patients bipolaires; b. Uniquement chez les patients traités par antipsychotiques ou neuroleptiques et en cas de manifestation compatible avec une hyperprolactinémie (aménorrhée, galactorrhée, baisse de la libido)

Concernant la place de l'IRM cérébrale, il est à noter que celle-ci peut être demandée sans urgence sauf dans les cas présentant une atypicité (voir infra) et pour l'EEG notre expérience montre qu'il peut être proposé dans les mêmes circonstances que l'IRM et que la présence d'anomalies mêmes parfois dites peu contributives (par exemple un léger ralentissement) orientent dans la plupart des cas vers une cause organique. De même, l'IRM cérébrale pourra conduire directement à un diagnostic (lésion frontale, encéphalite limbique, méningo-encéphalite à HSV1) ou justifier la recherche d'une cause inflammatoire et métabolique devant des anomalies aspécifiques (notamment des hypersignaux de la substance blanche ou une leucopathie).
} 
Si ce bilan semble déjà important, l'état actuel des recherches en psychiatrie montre qu'il est en réalité particulièrement insuffisant, notamment dans tous les cas présentant certaines atypicités, en cas de résistance aux traitements ou de pseudo-résistance.

\section{Bilan de $2^{\text {de }}$ intention : bilan des troubles pseudo-résistants}

La notion de résistance, qui se définit par rapport à un échec thérapeutique, ne prend pas en compte l'existence des facteurs complexes qui peuvent moduler la réponse indépendamment de tout traitement. Certains auteurs estiment ainsi que la résistance au traitement ne doit être évoquée que dans un contexte précis s'accompagnant d'un faisceau d'arguments solide et qu'en dehors de ces situations, il est préférable de parler de «pseudo-résistance » [9]. Après avoir éliminé un défaut d'observance, il est ainsi nécessaire d'évoquer :

1. L'erreur thérapeutique, qui consiste à traiter le patient avec un traitement inadapté à la pathologie (par exemple traiter un trouble anxiété généralisée avec des anxiolytiques).

2. L'erreur diagnostique, retrouvée dans les cas où le patient présente des symptômes typiques d'une pathologie psychiatrique alors qu'il s'agit en réalité d'un autre type de pathologie.

3. La résistance pharmacocinétique, lorsque le traitement est métabolisé trop rapidement pour pouvoir atteindre un taux thérapeutique.

4. La résistance pharmacodynamique, lorsque le traitement ne peut pas agir correctement sur sa cible, ou en cas d'absence de cofacteur ou de précurseurs permettant l'action du traitement.

Tableau 2. Résistance, pseudo-résistance et Atypicité

Résistance Absence d'amélioration significative des symptômes malgré deux lignes bien conduites de traitement issus des recommandations, à la posologie maximale tolérée/recommandée, pour une durée suffisante (4 à 6 semaines), en absence de cause de pseudo-résistance ou d'atypicités

Pseudo-résistance 1. Cas où la résistance est due à une erreur thérapeutique, une erreur diagnostique, une anomalie pharmacocinétique ou pharmacodynamique.

2. Cas ou la résistance est due à un facteur de médiation ou de modération entravant l'efficacité thérapeutique, par exemple une carence vitaminique ou une inflammation systémique.

Atypicités Ensemble de symptômes évocateurs d'un trouble organo-psychiatrique. 
Pour éviter une erreur thérapeutique, il est nécessaire de réévaluer le traitement dans toutes les situations de résistance, notamment en demandant un avis spécialisé si nécessaire. Certains patients traités depuis des années par Benzodiazépines pour un trouble anxieux bénéficient ainsi utilement d'un antidépresseur. Dans le même ordre d'idée, il est nécessaire de réévaluer les patients présentant un trouble schizo-affectif (association de symptômes schizophréniques et de symptômes bipolaires) traité par antipsychotiques seul (alors que l'adjonction d'un régulateur d'humeur classique serait appropriée) ou les patients bipolaires dépressifs traités par antidépresseur seuls. L'erreur diagnostique peut être d'ordre psychiatrique (considérer un trouble dépressif bipolaire pour un trouble dépressif unipolaire, considérer un trouble anxieux sévère pour un trouble psychotique, etc.) ou d'ordre somatique. Concernant la résistance pharmacocinétique, la recherche d'un profil particulier de métabolisation des traitements peut s'envisager lorsque les taux plasmatiques ne sont pas concordants avec les posologies prescrites malgré une observance correcte. Chez ces patients un avis pharmacogénétique est nécessaire avant la réintroduction d'un traitement psychotrope. En ce qui concerne certains facteurs de médiation, et notamment en cas de carence vitaminique, il apparaît de plus en plus clairement que les seuils actuels, qui ont été défini par rapport à des pathologies neurologiques ou hématologiques, ne sont pas adaptés aux patients psychiatriques, et que de nouveaux seuils mériteraient d'être élaborés comme cela a déjà été fait avec les folates dont l'insuffisance (folatémie $<10 \mathrm{ng} / \mathrm{ml}$ ) est fortement corrélée à l'inefficacité des traitements antidépresseurs ou à la persistance de symptômes dépressifs. Les marqueurs périphériques d'inflammation telle que la CRP sont aussi corrélés à la pseudo-résistance et à l'intensité des symptômes dépressifs, y compris à des taux considérés comme non pathologiques mais proche de la limite supérieure des valeurs normales [11]. Il en va de même pour les variations de la TSH chez les patients dysthydroïdiens habitués à avoir toujours le même taux et dont on sait que des augmentations même minimes (et restant dans les normes physiologiques) sont corrélées à la survenue de troubles dépressifs qui se corrigent après adaptation du traitement [16]. C'est aussi le cas de certains patients présentant des hyponatrémies (parfois assez peu profondes, $128+/-3 \mathrm{mEq} / \mathrm{L}$ ) mais qui vont se manifester par d'importants troubles du comportement ou une agitation qui va faire poser à tort un diagnostic psychiatrique alors qu'une correction ionique permettrai de faire céder les symptômes psychiatriques [10]. Enfin, pour ce qui est de la résistance pharmacodynamique, la prise en 
compte du profil pharmacologique de la molécule est nécessaire. Ainsi proposer un inhibiteur de la recapture de la sérotonine alors que le patient présente un état inflammatoire ou une carence vitaminique majeure entraînant une diminution des précurseurs de la sérotonine a peu de sens (comme en atteste l'absence d'efficacité de ces molécules chez les patients ayant un cancer actif). Il en est de même concernant l'association de molécules ayant des propriétés antagonistes qui risquent de mettre en échec le traitement (par exemple traiter une dépression avec un bloqueur dopaminergique fort, ou associer agoniste et antagoniste dopaminergique). Nous proposons dans le tableau 2 un bilan de pseudo-résistance. 
Tableau 3. Bilan de pseudo-résistance ( $2^{\text {nd }}$ intention $)$

\section{Recherche de médiateurs organo-psychiatriques}

CRP ultrasensible [11] Une inflammation systémique, même minime (CRP entre 3 et $10 \mathrm{mg} / \mathrm{l})$ et persistante en absence de facteur explicatif, doit être recherchée car il s'agit d'un facteur de stress cérébral qui va péjorer les symptômes (notamment dépressifs). Un syndrome psychiatrique non contrôlé associé à une élévation chronique de la CRP (> $5 \mathrm{mg} / \mathrm{l})$ doit faire demander un avis spécialisé (bilan infectieux et auto-immunitaire)

Folates [12]

La vitamine B9 (folates) est impliquée dans la méthylation de l'ADN, fait partit du cycle de l'homocystéine et est un précurseur de la sérotonine. Une carence en folates (définie par une folatémie $9 \mathrm{nmol} / \mathrm{L}$ ou $4 \mathrm{ng} / \mathrm{ml}$ ) est associée à un risque accru de dépression, mais même une insuffisance (définie par une folatémie $<22 \mathrm{nmol} / \mathrm{L}$ ou $10 \mathrm{ng} / \mathrm{ml}$ ) augmente significativement le risque et nécessite une supplémentation adaptée.

Vitamine B12 [13, 14] La vitamine B12 est un cofacteur des folates qui intervient dans le cycle de l'homocystéine. Une carence en vitamine B12 peut se manifester par une symptomatologie psychiatrique parfois plusieurs années avant l'émergence des signes hématologiques ou neurologiques. Sur le plan dépressif, un taux bas de vitamine B12 est corrélé à la dépression essentiellement chez les femmes.

Homocystéine [15]

Une hyperhomocystéinémie altère les processus de méthylation qui sont nécessaire pour la synthèse de nombreuses monoamines dont les déficits sont impliqués dans la physiopathologie des maladies mentales. Une partie des effets délétères de l'hyperhomocystéinémie passerait par l'action particulière de la S-adénosylhomocystéine sur la catéchol-O-méthyltransférase (COMT). Il est aussi démontré que l'homocystéine avait des propriétés d'antagoniste partiel sur le site glycine des récepteurs NMDA : lorsque les concentrations de glycine augmentent (par exemple lors d'une AVC où l'homocystéine et la glycine passent la barrière hématoencéphalique) l'homocystéine devient cytotoxique par activation excessive (effet agoniste) des NMDA. Par ailleurs, l'hyperhomocystéïnémie peut être le reflet d'un trouble du métabolisme ou d'une carence en vitamine B12, B9 ou B6.

TSH [16] En cas de syndrome dépressif (surtout bipolaire), plusieurs études récentes suggèrent qu'une TSH $>2,5$ voire même $2 \mathrm{mU} / \mathrm{L}$ est associée avec une absence de réponse au traitement. D'un point de vue thérapeutique, cela permet de proposer plus facilement des stratégies de potentialisation aux hormones thyroïdienne dans ces cas-là. 


\begin{abstract}
Dosage plasmatique Un dosage plasmatique en résiduel (c'est-à-dire juste avant la prise du traitement) est [17] nécessaire pour s'assurer d'une part que le patient est observant et d'autre part que les taux plasmatiques des médicaments sont suffisant pour permettre une efficacité thérapeutique. Il est à noter que l'HAS recommande un tel dosage en cas d'inefficacité de la thérapeutique employée.
\end{abstract}

Pharmacogénétique

(à proposer en cas d'anomalie sur le dosage plasmatique) [18]
Après avoir éliminé un défaut d'observance ou une interaction médicamenteuse (avec un inducteur enzymatique), il est possible de demander un séquençage génétique permettant la détection des métaboliseurs rapide (MR) ou ultra-rapides (MUR) pour les cytochromes CYP 3A4, 2D6 et 2C19, les principaux cytochromes impliqués dans la métabolisation des psychotropes (antidépresseurs et antipsychotiques notamment). Cette approche permet une pharmacothérapie personnalisée.

Nous pouvons également citer deux médiateurs de plus en plus étudiés : l'influence des lipides (acides gras, sphingolipides, stéroïdes, etc.) sur les symptômes dépressifs et psychotiques [19], et l'impact des dysfonctions mitochondriales (stress oxydatif, dysrégulation métabolique et hormonale, modification de la perméabilité membranaire, etc.) sur les maladies neuropsychiatriques [20]

\title{
4. Bilan de $3^{\text {ème }}$ intention : Bilan d'atypicité
}

Concernant essentiellement les troubles schizophréniques et les syndromes catatoniques, les atypicités doivent être recherchées devant tout syndrome psychiatrique qui ne répond pas correctement aux traitements. Elles ont été caractérisées par les équipes de Frédéric Sedel, Olivier Bonnot et David Cohen (Pitié-Salpêtrière) [21, 22] qui ont proposé un regroupement sous la forme de trois situations :

1. Les situations d'urgence où les troubles peuvent se manifester sous forme de syndromes confusionnels aigus et récurrents, avec un risque de diagnostic erroné de psychose aiguë ou de manie atypique.

2. Les atypicités neuropsychiatriques (tableau 3) lorsque les troubles sont caractérisés par la présence de signes tels que des hallucinations visuelles ou de la catatonie, avec une 
potentielle aggravation si des médicaments antipsychotiques ou neuroleptiques sont introduits

3. Les maladies caractérisées par un léger retard mental et des changements tardifs de comportement ou de personnalité.

Tableau 3. Atypicités neuropsychiatriques

\section{Adapté de Bonnot $O$ et al.}

Hallucinations visuelles

Catatonie

Symptômes cognitifs d'évolution progressive / retard mental

Fluctuation des symptômes

Effets indésirables fréquents ou sévères des traitements

Symptomatologie confusionnelle

Symptômes neurologiques «mineurs »: tremblements, perte d'équilibre, rigidité, anomalies motrice, mouvements anormaux, neuropathie

Symptômes neurologiques « majeurs » : épilepsie

Symptômes digestifs : crises de douleurs abdominales, modification du régime alimentaire

Début aigu ou précoce

\section{Autres signes évocateurs}

Trouble mnésiques antérogrades et/ou rétrogrades

Altération persistante des fonctions cognitives sur des tests (TMT-A, TMT-B, MoCA)

Syndrome pseudo-grippal peu de temps avant l'apparition des troubles psychiatriques

Concernant les troubles dépressifs, outre les classiques endocrinopathies (hypothyroïdie, hypo ou hypercorticisme, etc.), il est peu fait mention d'atypicités (au sens organique) dans la littérature, mais 
certaines pathologies (essentiellement de surcharge et auto-immunes) peuvent être pourvoyeuses de troubles.

Dans toutes ces situations, il peut être nécessaire de procéder à des explorations plus larges, possiblement en unité spécialisée, avec pour objectif la recherche d'un certain nombre de pathologies organiques d'expression psychiatrique qui peuvent être regroupées en plusieurs grandes classes (tableau 5) : 1. Les maladies infectieuses, 2. les maladies auto-immunes et inflammatoires, 3. les maladies neuro-dégénératives, 4. les épilepsies, 5. les maladies neuro-métaboliques et maladies de surcharge, 6 . les maladies enzymatiques, 7. les causes toxiques (qui seront abordée dans le $2^{\text {nd }}$ volet de cet article). Ces maladies sont abordées ici avec les principaux symptômes psychiatriques, physiques et examens complémentaires nécessaires, et seront décrites plus en détail dans le second volet du présent article. Il est à noter que les signes et symptômes rapportés dans ce tableau sont de fréquence variable, le tableau pouvant s'exprimer - dans de rares cas - par des symptômes psychiatriques isolés sans aucun symptôme organique associé ou avec des symptômes organiques évoluant à bas bruit et non repérés jusqu'alors. Les symptômes organiques rapportés ici sont les plus fréquents, certains symptômes rares peuvent manquer mais seront détaillés dans le second volet du présent article. Il convient aussi de noter que les examens proposés peuvent être négatifs dans un certain nombre de cas, à titre d'exemple, dans l'encéphalite limbique à anti-NMDAR l'IRM cérébrale est normale dans 65\% des cas et l'EEG est normal dans environ $20 \%$ des cas [23] : une absence de signe organique ou la normalité d'un examen complémentaire ne doit donc pas faire écarter le diagnostic.

Tableau 5. Pathologies organo-psychiatriques

\begin{tabular}{lllc}
\hline Pathologies & $\begin{array}{l}\text { Symptômes } \\
\text { Psychiatriques }\end{array}$ & $\begin{array}{l}\text { Symptômes } \\
\text { organiques }\end{array}$ & Examens diagnostics \\
& \multicolumn{2}{c}{ Maladies infectieuses } \\
\hline $\begin{array}{l}\text { Méningo-encéphalite } \\
\text { herpétique [24] }\end{array}$ & Episode délirant aigu & Fièvre (rarement fébricule) & PL avec PCR HSV1 \\
& Syndrome catatonique & $\begin{array}{l}\text { Céphalées } \\
\text { Confusion }\end{array}$ & $\begin{array}{c}\text { IRM (atteinte temporale) } \\
\text { EEG (ondes lentes } \\
\text { pseudo-périodiques } \\
\text { temporales) }\end{array}$ \\
\hline
\end{tabular}




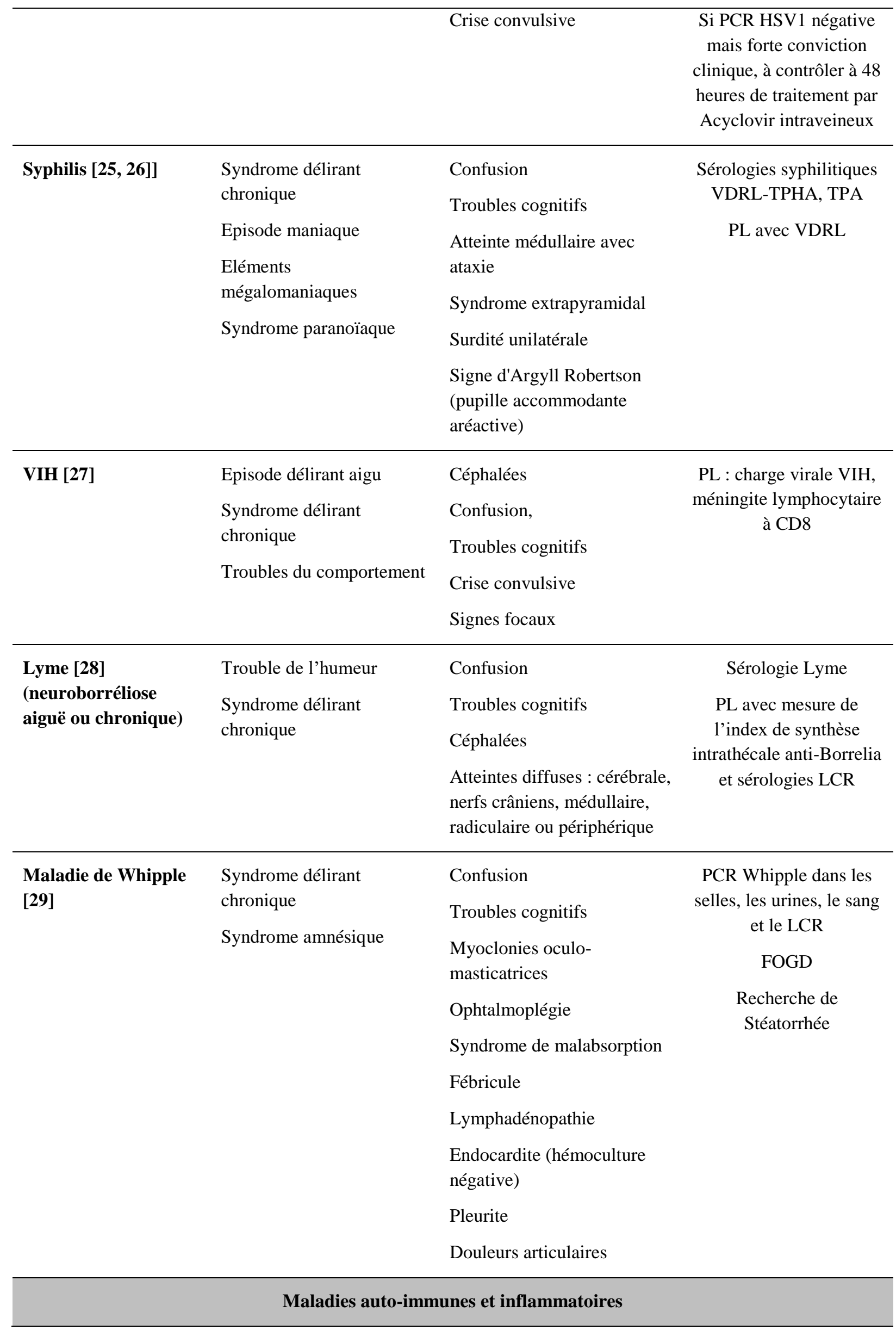




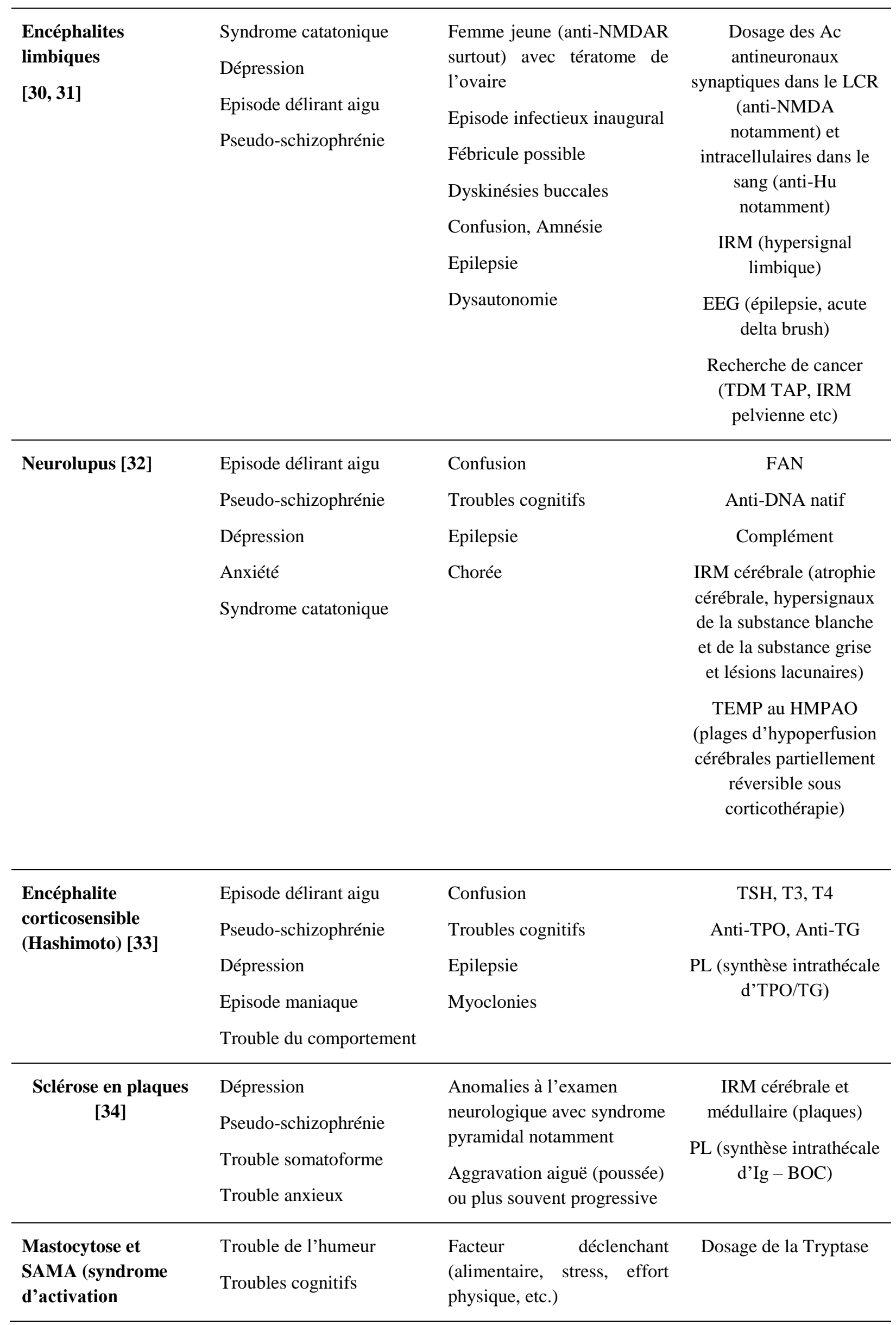




\begin{tabular}{|c|c|c|c|}
\hline \multirow[t]{7}{*}{ mastocytaire) [35] } & Troubles anxieux & Urticaire / Dermographisme & \\
\hline & \multirow[t]{6}{*}{ Troubles somatoforme } & Dysurie & \\
\hline & & Flush & \\
\hline & & Intolérance alimentaire & \\
\hline & & $\begin{array}{l}\text { Antécédent de choc } \\
\text { anaphylactique ou d'œdème } \\
\text { de Quinck }\end{array}$ & \\
\hline & & Céphalées & \\
\hline & & Flou visuel & \\
\hline \multicolumn{4}{|c|}{ Maladies neuro-dégénératives } \\
\hline \multirow{5}{*}{$\begin{array}{l}\text { Maladie d'Alzheimer } \\
{[36]}\end{array}$} & \multirow{3}{*}{$\begin{array}{l}\text { Dépression } \\
\text { Trouble psychotique (type } \\
\text { paranoïa) }\end{array}$} & \multirow{5}{*}{$\begin{array}{l}\text { Age }>50 \text { ans } \\
\text { Trouble de la mémoire } \\
\text { épisodique }\end{array}$} & IRM (atrophie \\
\hline & & & hippocampique) \\
\hline & & & Scintigraphie cérébrale \\
\hline & \multirow[t]{2}{*}{ Hallucinations (rare) } & & $\begin{array}{l}\text { (hypometabolisme } \\
\text { temporal interne) }\end{array}$ \\
\hline & & & $\begin{array}{l}\text { PL (biomarqueurs de } \\
\text { neurodégénérescence) }\end{array}$ \\
\hline \multirow{3}{*}{$\begin{array}{l}\text { Maladie de Parkinson } \\
\text { [37] }\end{array}$} & Dépression & Age $>50$ ans & Essentiellement clinique \\
\hline & Trouble délirant & Syndrome parkinsonien & Si doute : DAT-scan \\
\hline & $\begin{array}{l}\text { Hypomanie et trouble du } \\
\text { contrôle des impulsions } \\
\text { (en lien avec le traitement } \\
\text { par agoniste } \\
\text { dopaminergique) }\end{array}$ & & \\
\hline \multirow{7}{*}{$\begin{array}{l}\text { Maladie à corps de } \\
\text { Lewy diffus [38] }\end{array}$} & \multirow{7}{*}{$\begin{array}{l}\text { Trouble psychotique } \\
\text { hallucinatoire } \\
\text { Dépression }\end{array}$} & Age $>50$ ans & Clinique \\
\hline & & Déclin cognitif & Si doute : DAT-scan et \\
\hline & & Syndrome parkinsonien & $\begin{array}{l}\text { Scintigraphie cérébrale } \\
\text { (hypoperfusion pariéto- }\end{array}$ \\
\hline & & Fluctuations & occipital) \\
\hline & & Troubles neurovégétatifs & \\
\hline & & Hallucinations visuelles & \\
\hline & & Episodes confusionnels & \\
\hline \multirow{5}{*}{$\begin{array}{l}\text { Dégénérescence } \\
\text { fronto-temporale [39] }\end{array}$} & Dépression & Age $>50$ ans & \multirow{2}{*}{$\begin{array}{l}\text { IRM (atrophie frontale } \\
\text { et/ou temporale) }\end{array}$} \\
\hline & Hypomanie & Apathie sans tristesse & \\
\hline & $\begin{array}{l}\text { Modification de la } \\
\text { personnalité }\end{array}$ & $\begin{array}{l}\text { Syndrome dysexécutif } \\
\text { Syndrome frontal }\end{array}$ & $\begin{array}{c}\text { Dosage plasmatique de } \\
\text { la progranuline }(+/- \\
\text { recherche génétique) }\end{array}$ \\
\hline & \multirow[t]{2}{*}{$\begin{array}{l}\text { Trouble du comportement } \\
\text { alimentaire }\end{array}$} & $\begin{array}{l}\text { comportemental (grasping, } \\
\text { désinhibition notamment) }\end{array}$ & $\begin{array}{l}\text { Si doute : Scintigraphie } \\
\text { cérébrale (hypoperfusion }\end{array}$ \\
\hline & & Démence & frontale et/ou temporale) \\
\hline
\end{tabular}




\section{« Neurologiques »}

\begin{tabular}{|c|c|c|c|}
\hline \multirow[t]{2}{*}{ Epilepsie frontale 40} & \multirow{2}{*}{$\begin{array}{l}\text { Trouble du comportement } \\
\text { paroxystique }\end{array}$} & \multirow{3}{*}{$\begin{array}{l}\text { Hallucinations riches } \\
\text { Critique partielle } \\
\text { Syndrome post-ictal }\end{array}$} & \multirow{5}{*}{$\begin{array}{c}\text { EEG } \\
\text { IRM cérébrale } \\
\text { Holter-EEG, EEG de } \\
\text { privation de sommeil } \\
\text { Vidéo-EEG }\end{array}$} \\
\hline & & & \\
\hline \multirow{2}{*}{$\begin{array}{l}\text { Epilepsie temporale } \\
41\end{array}$} & Psychose hallucinatoire & & \\
\hline & Hallucinations olfactives & & \\
\hline Epilepsie occipitale & Hallucinations visuelles & & \\
\hline \multirow{4}{*}{$\begin{array}{l}\text { Tumeur cérébrale } \\
\text { [42] }\end{array}$} & Dépression & Troubles mnésiques & \multirow{4}{*}{$\begin{array}{c}\text { IRM (processus } \\
\text { occupant de l'espace) }\end{array}$} \\
\hline & Psychose & Syndrome frontal & \\
\hline & Anxiété & Epilepsie & \\
\hline & $\begin{array}{l}\text { Troubles du comportement } \\
\text { alimentaire }\end{array}$ & & \\
\hline
\end{tabular}

\begin{tabular}{|c|c|c|c|}
\hline \multicolumn{4}{|c|}{ Maladies endocriniennes } \\
\hline \multirow{9}{*}{$\begin{array}{c}\text { Insuffisance } \\
\text { surrénalienne [43] }\end{array}$} & Dépression & Asthénie & Ionogramme \\
\hline & Psychose & Amaigrissement & $\begin{array}{l}\text { (Hyponatrémie, } \\
\text { Hyperkaliémie) }\end{array}$ \\
\hline & Manie & Hypotension & Glycémie \\
\hline & Anxiété & Malaises & (Hypoglycémie) \\
\hline & Hallucinations & Mélanodermie & Cortisolémie au réveil \\
\hline & & Vitiligo & Test au Synacthène \\
\hline & & Troubles mnésiques & \\
\hline & & Confusion & \\
\hline & & Troubles digestifs & \\
\hline \multirow{6}{*}{$\begin{array}{c}\text { Phéochromocytome } \\
{[44]}\end{array}$} & Trouble panique & HTA & Dosage répété des \\
\hline & Trouble dépressif & Triade de Ménard : & $\begin{array}{c}\text { catécholamines urinaires } \\
\text { (Métanéphrine et }\end{array}$ \\
\hline & & - Céphalées & Normétanéphrine urinaire \\
\hline & & - Palpitations & des 24 h augmenté) \\
\hline & & - Sueurs & TDM-TAP \\
\hline & & & +/- Scintigraphie MIBI \\
\hline \multicolumn{4}{|c|}{ Maladie neurométaboliques et maladies de surcharge } \\
\hline \multirow{5}{*}{$\begin{array}{l}\text { Déficit cérébral en } \\
\text { folates [45] et carence } \\
\text { intracérébrale en } \\
\text { folates par auto- } \\
\text { anticorps anti-FRa } \\
\text { [46] }\end{array}$} & Pseudo-schizophrénie & Ataxie & Folates sanguins \\
\hline & Pseudo-autisme & Syndrome pyramidal & Dosage des folates et du \\
\hline & Dépression & Dyskinésies & $\begin{array}{l}\text { 5MTHF (5- } \\
\text { methyltétrahydrofolate) }\end{array}$ \\
\hline & Troubles cognitifs & Crise convulsive & dans le LCR (recherche \\
\hline & Troubles du sommeil & Troubles cognitifs & $\begin{array}{l}\text { de carence cérébrale en } \\
\text { folate avec folatémie }\end{array}$ \\
\hline
\end{tabular}




\begin{tabular}{|c|c|c|c|}
\hline & & & $\begin{array}{c}\text { normale) } \\
\text { Ac Anti-FR } \alpha \text { (sérum) } \\
\text { IRM (anomalies sur la } \\
\text { séquence de } \\
\text { spectroscopie) }\end{array}$ \\
\hline $\begin{array}{l}\text { Déficit en vitamine } \\
\text { B12 [47] }\end{array}$ & $\begin{array}{l}\text { Pseudo-schizophrénie } \\
\text { Pseudo-autisme } \\
\text { Dépression } \\
\text { Troubles cognitifs } \\
\text { Troubles du sommeil }\end{array}$ & $\begin{array}{l}\text { Anémie macrocytaire } \\
\text { Glossite } \\
\text { Troubles cognitifs } \\
\text { Sclérose combinée de la } \\
\text { moelle / ataxie } \\
\text { proprioceptive } \\
\text { Troubles sphinctériens }\end{array}$ & $\begin{array}{c}\text { Vitamine B12, } \\
\text { Homocystéinémie, } \\
\text { Acide méthylmalonique } \\
\text { Ac Anti-Facteur } \\
\text { intrinsèque - cellules } \\
\text { pariétales }\end{array}$ \\
\hline $\begin{array}{l}\text { Niemann Pick type C } \\
{[48]}\end{array}$ & $\begin{array}{l}\text { Hallucination } \\
\text { Désorganisation } \\
\text { Pseudo-schizophrénie } \\
\text { Pseudo-autisme }\end{array}$ & $\begin{array}{l}\text { Ophtalmolplégie } \\
\text { supranucléaire } \\
\text { Ataxie } \\
\text { Dystonie } \\
\text { Dysarthrie } \\
\text { Troubles cognitifs } \\
\text { Epilepsie } \\
\text { Organomégalie }\end{array}$ & $\begin{array}{c}\text { Dosage des Oxystérols } \\
\text { et chitotriosidase } \\
\text { plasmatique } \\
\text { Biopsie de peau pour } \\
\text { culture de fibroblaste } \\
\text { Recherche des mutations } \\
\text { NPCl et } 2\end{array}$ \\
\hline $\begin{array}{l}\text { Xanthomatose } \\
\text { cérébro-tendineuse } \\
\text { [49] }\end{array}$ & $\begin{array}{l}\text { Pseudo-schizophrénie } \\
\text { Trouble de l'humeur } \\
\text { Syndrome suicidaire }\end{array}$ & $\begin{array}{l}\text { Xanthome tendineux } \\
\text { Epilepsie } \\
\text { Ataxie cérébelleuse } \\
\text { Syndrome parkinsonien } \\
\text { Diarrhée chronique } \\
\text { Cataracte }\end{array}$ & $\begin{array}{l}\text { Cholestanol plasmatique } \\
\text { Hypocholestérolémie } \\
\text { IRM (leucopathie, } \\
\text { hypersignal des noyaux } \\
\text { dentelés) }\end{array}$ \\
\hline $\begin{array}{l}\text { Maladie de Wilson } \\
{[50]}\end{array}$ & $\begin{array}{l}\text { Dépression } \\
\text { Pseudo-schizophrénie } \\
\text { Episode délirant aigu } \\
\text { Labilité thymique } \\
\text { Irritabilité, Anxiété } \\
\text { Trouble du comportement } \\
\text { (bizarreries inexpliqués, ex } \\
\text { : phobies soudaines) } \\
\text { Difficultés attentionnelle, } \\
\text { mnésique, concentration }\end{array}$ & $\begin{array}{l}\text { Syndrome extrapyramidal } \\
\text { Dystonie faciale } \\
\text { Troubles cognitifs } \\
\text { Anneau de Kayser Fleicher } \\
\text { (examen à la lampe à fente) } \\
\text { Hépatopathie sévère } \\
\text { Atteinte hormonale sexuelle }\end{array}$ & $\begin{array}{c}\text { Cuprémie } \\
\text { Cuprurie } \\
\text { Céruloplasmine } \\
\text { IRM (signe du panda) } \\
\text { Explorations hépatiques }\end{array}$ \\
\hline
\end{tabular}




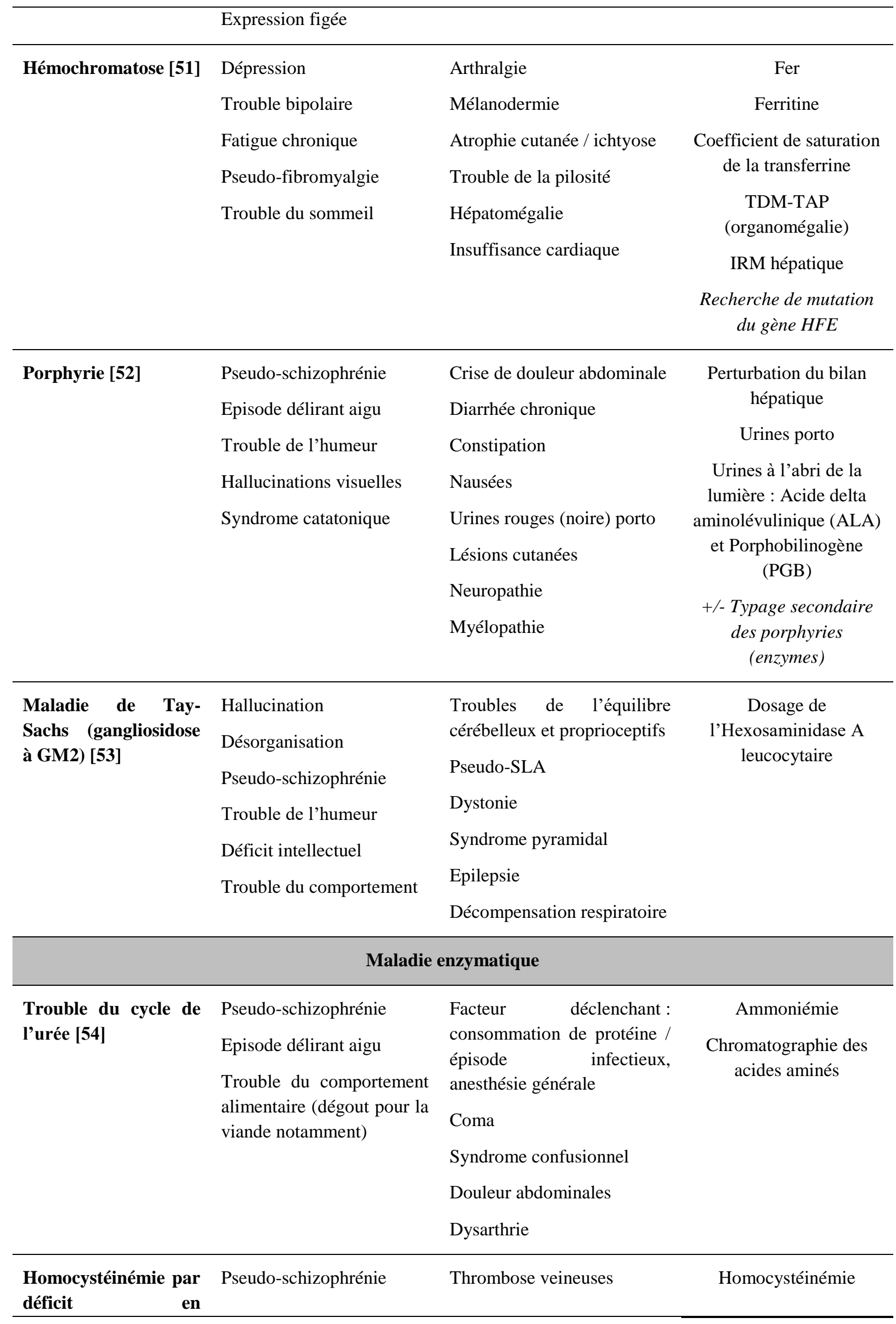




\begin{tabular}{|c|c|c|c|}
\hline \multirow{4}{*}{$\begin{array}{l}\text { cystathionine betha- } \\
\text { synthase (CBS) [15, } \\
\text { 55] }\end{array}$} & Episode délirant aigu & Aspect marfanoïde & Homocystéinurie \\
\hline & TOC & Scoliose & Dosage de la Méthionine \\
\hline & Troubles de l'humeur & Myopie, ectopie cristallin & ETT (recherche \\
\hline & & Syndrome cérébelleux & d'anomalies aortiques) \\
\hline \multirow{3}{*}{$\begin{array}{l}\text { Homocystéinémie par } \\
\text { déficit en MTHFR } \\
\text { [56] }\end{array}$} & Pseudo-schizophrénie & Apnées & Doly rmmorabi \\
\hline & Episode délirant aigu & Epilepsie & romysominograpine \\
\hline & Hallucinations visuelles & Ataxie & \\
\hline \multirow[t]{4}{*}{ Phénylcétonurie [57] } & Hyperactivité & Epilepsie & Phénylalanine sanguine \\
\hline & Anxiété & Tremblement & Déficit en \\
\hline & Dépression & Retard mental & $\begin{array}{l}\text { phénylalanine } \\
\text { hydroxylase }\end{array}$ \\
\hline & & Troubles cognitifs & \\
\hline
\end{tabular}

\section{Discussion. Le concept de troubles psychiatriques complexes}

Un système complexe peut être défini par l'existence de relations causales non linéaires comportant différentes niveaux d'organisation et des boucles de rétroaction [58]. De ce fait, ces systèmes sont caractérisés par un grand nombre de niveau d'observation et d'interprétation - qui se recoupent partiellement - pour aboutir à un ensemble cohérent de composants en interaction. Les troubles psychiatriques regroupent justement un ensemble très hétérogène de pathologies dont la variabilité physio-psycho-pathologique est importante. Le concept de trouble psychiatrique complexe que nous proposons ici, recouvre un ensemble de situations marquées par une grande variabilité clinique. De tels troubles peuvent avoir une cause unique mais le plus souvent il existe une ou plusieurs « chaînes causales » qui peuvent impliquer des facteurs de risque génétiques, environnementaux, sociaux et biologiques. L'effet d'un de ces facteurs de risque ne peut être pleinement compris sauf si on l'intègre dans un ensemble plus vaste. De facto, il est nécessaire de s'intéresser aux concepts de médiateurs (variables intermédiaires), de modérateurs (modificateurs d'effets) et d'interactions réciproques [59]. De nombreuses études en psychologie et en épidémiologie s'y sont déjà intéressées et il semble opportun de permettre à la psychiatrie de bénéficier de ce type d'approche.

On distingue ainsi : 
- Les facteurs de modération, qui regroupent les facteurs qui agissent directement sur un élément en vue d'en modifier certaines caractéristiques. C'est par exemple le cas de certains génotypes dont on sait qu'ils vont influencer directement l'efficacité de certaines drogues ou médicaments. Ainsi un polymorphisme du gène du transporteur de la sérotonine va modérer l'efficacité de certains antidépresseurs. Un autre exemple concerne l'homocystéine, un acide aminé résultant du catabolisme de la méthionine, dont on sait que l'action au niveau cérébrale va être neurotoxique et qui va favoriser la résistance aux traitements en diminuant le nombre de neurotransmetteurs.

- Les facteurs de médiation, qui peuvent être définis comme les facteurs qui influencent de manière indirecte la façon dont un élément va agir sur un autre. Par exemple, une corrélation entre toxoplasmose et schizophrénie a régulièrement été mise en évidence dans plusieurs études sans qu'un lien de causalité franc ait pu être retrouvé. L'une des explications possible est que chez des patients présentant une vulnérabilité, l'inflammation cérébrale à bas bruit que peut générer une inflammation systémique va agir comme un puissant agent déclencheur.

- Les facteurs d'interaction réciproque, pour lesquels le sens de la causalité est bidirectionnel comme c'est par exemple le cas lorsque l'on étudie les liens entre maladies auto-immunes et psychiatrie. Les maladies auto-immunes ont une action sur le déclenchement de certains troubles psychiatriques (psychotiques ou thymiques) et à l'inverse les maladies psychiatriques vont avoir une influence sur des mécanismes immunologiques via les modifications aminergiques ou gabaergiques qu'elles entraînent, soit en augmentant la réaction inflammatoire via l'axe du stress, soit via un effet immunodépressogène.

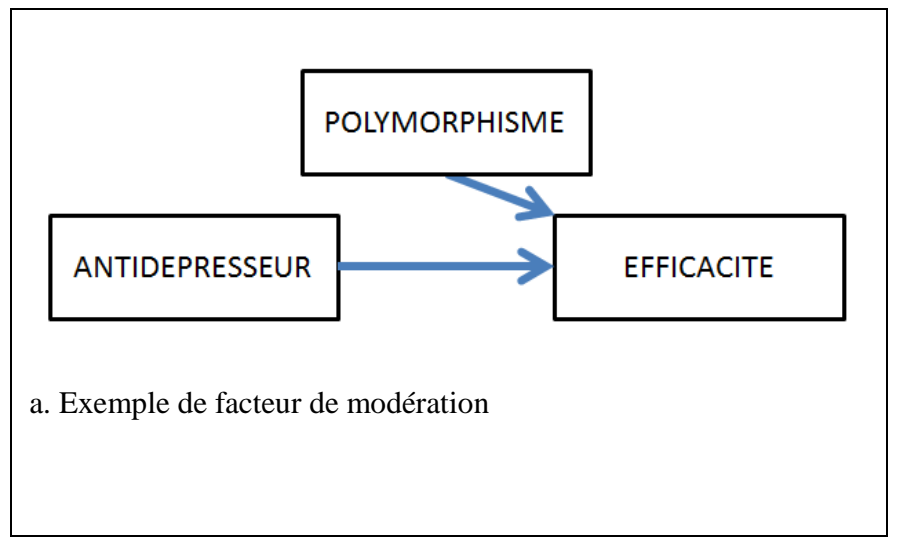




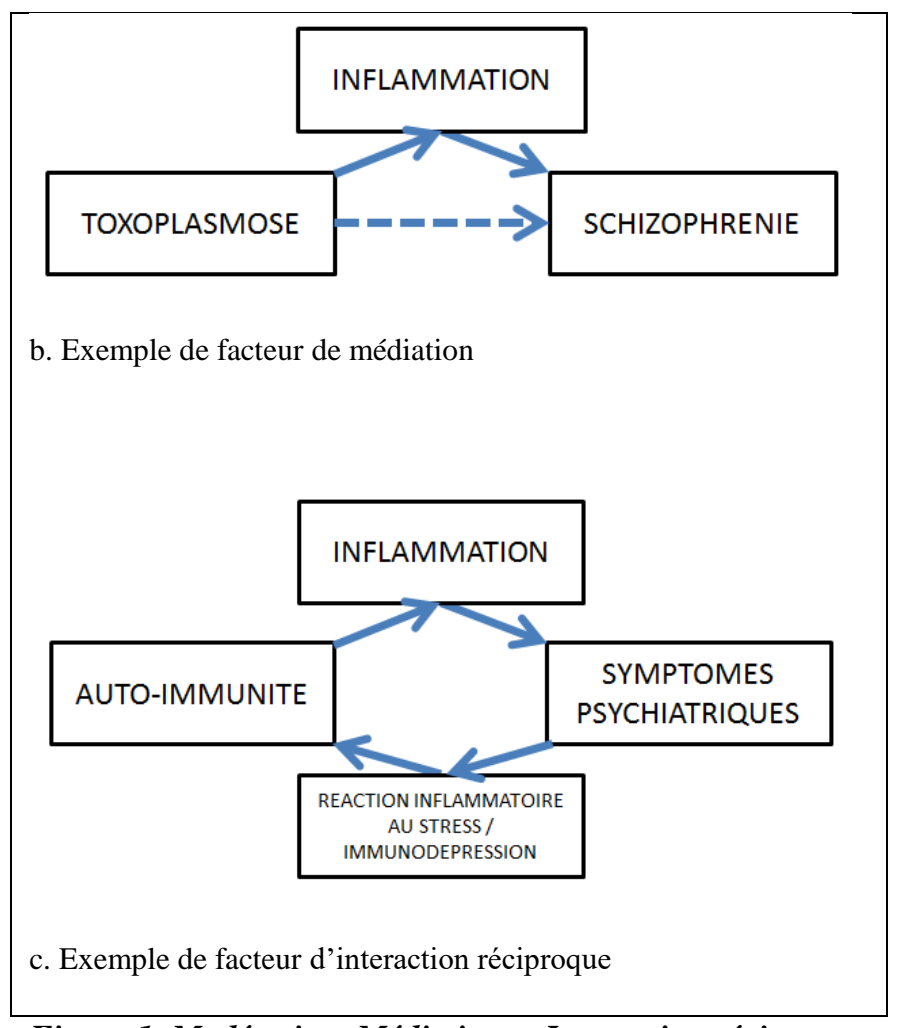

Figure 1. Modération, Médiation et Interaction réciproque

La prise en compte de ces différents niveaux d'interaction entre des éléments organiques et psychiatriques permet d'ouvrir un nouveau champ de réflexion pour la prise en charge de ces troubles qui varient en fréquence et en intensité.

Il en résulte parfois une certaine «atypicité » des troubles psychiatriques complexe, soit du fait de l'existence de modulation phénotypique pouvant entraîner l'apparition de signes psychiatriques rares ou même d'allure neurologique, soit du fait de la résistance aux traitements que cela peut entrainer. Il apparaît ainsi nécessaire de s'intéresser aux facteurs inflammatoires, nutritionnels (vitaminiques notamment), génétiques, métaboliques et endocriniens qui peuvent s'intégrer dans le cadre d'un bilan de «pseudo-résistance » et d'atypicité. 


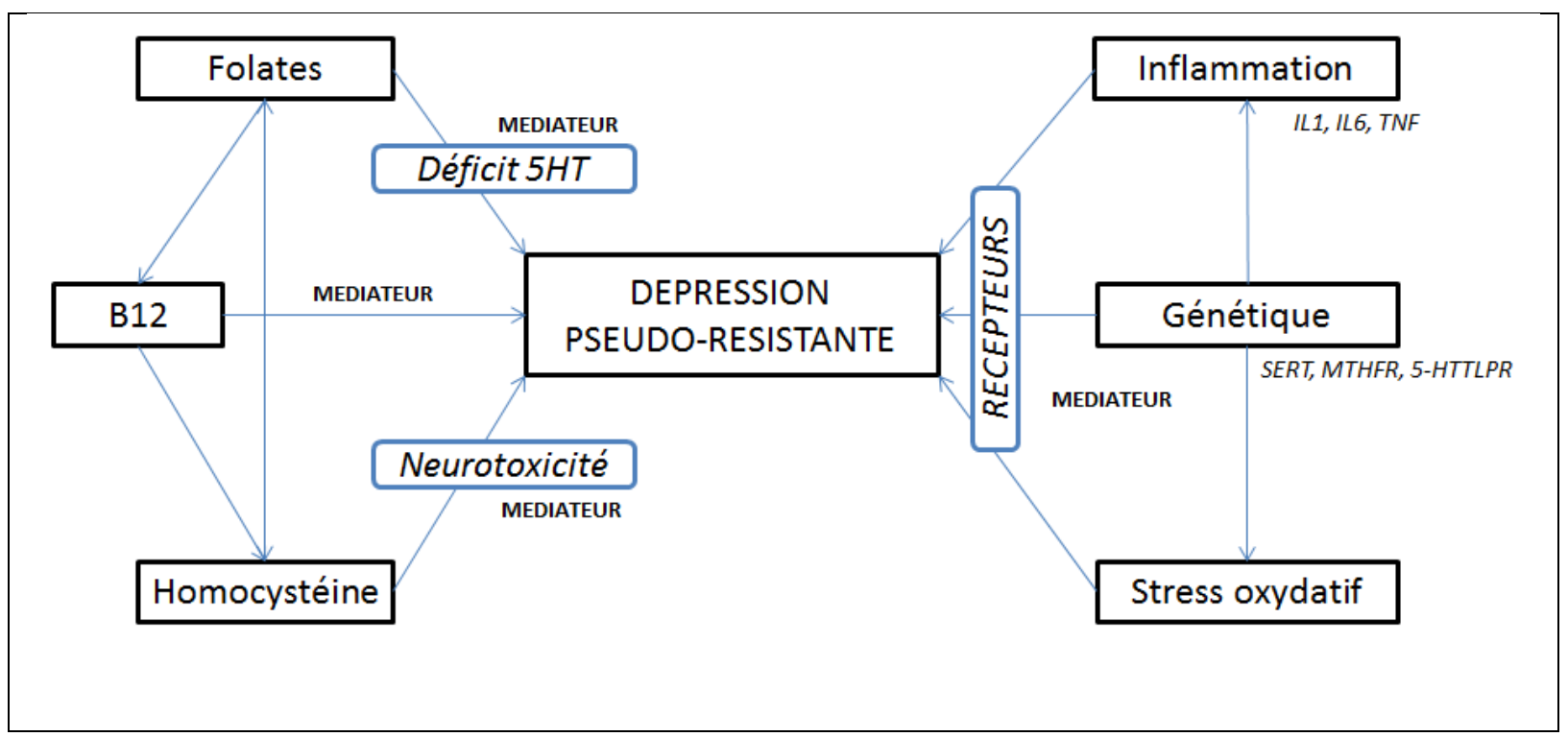

Figure 2. Exemple de médiateurs appliqué à la dépression

On pourrait opposer à ce type de bilan qui semble complexe et invasif la question du rapport coûtbénéfice, et l'on pourrait même introduire la notion de « Nombre de Patient à Explorer » (une version diagnostique du NNT - Number Needed to Treat - en quelque sorte) pour diagnostiquer une pathologie organique curable en lieu et place d'un trouble psychiatrique chronique. Un rapport de la CNAM en 2016 retrouve que 14,1 milliards d'euros sont affectés annuellement aux pathologies psychiatriques et que près de $70 \%$ de ces dépenses sont répartis sur les « troubles névrotiques ou de l'humeur » et les « troubles psychotiques » [60]. Parmi ces patients, le dépistage et la prise en charge adaptée des «faux-positifs » psychiatriques permettraient de réduire une partie de ces coûts, et des études médico-économiques sont nécessaires pour en déterminer l'importance.

Les modalités de la collaboration (réunion multidisciplinaire, bilan systématisé, parcours patient adapté, spécialiste ressource, etc.) entre les différentes spécialités confrontées à ces troubles sera abordé dans une seconde partie, mais ce type de coopération semble de plus en plus indispensable à l'heure où de nombreux services de psychiatrie sont mis à distance des plateaux techniques spécialisés, avec d'importantes conséquences en matière de retard diagnostic et de perte de chance thérapeutique pour près de $10 \%$ de nos patients. De même, le rationnel pathologie par pathologie, la fréquence des signes cliniques et des anomalies retrouvés sur les examens complémentaires, les opportunités thérapeutiques qui en découlent, une proposition de démarche standardisée seront 
proposés tandis que le caractère invasif et le coût de ce type de bilan seront discutés dans le second volet du présent article.

\section{Références}

1. Deacon BJ. The biomedical model of mental disorder: a critical analysis of its validity, utility, and effects on psychotherapy research. Clin Psychol Rev. 2013 Nov;33(7):846-61. doi: 10.1016/j.cpr.2012.09.007.

2. Testa A, Giannuzzi R, Daini S, Bernardini L, Petrongolo L, Gentiloni Silveri N. Psychiatric emergencies (part III): psychiatric symptoms resulting from organic diseases. Eur Rev Med Pharmacol Sci. 2013 Feb;17 Suppl 1:86-99. Review. PubMed PMID: 23436670.

3. Talbot-Stern JK, Green T, Royle TJ. Psychiatric manifestations of systemic illness. Emerg Med Clin North Am. 2000 May;18(2):199-209, vii-viii. Review. PubMed PMID: 10767878.

4. Hall RC, Popkin MK, Devaul RA, Faillace LA, Stickney SK. Physical illness presenting as psychiatric disease. Arch Gen Psychiatry. 1978 Nov;35(11):1315-20. PubMed PMID: 568461.

5. Johnstone EC, Macmillan JF, Crow TJ. The occurrence of organic disease of possible or probable aetiological significance in a population of 268 cases of first episode schizophrenia. Psychol Med. 1987 May;17(2):371-9. PubMed PMID 3602229.

6. Reeves RR, Pendarvis EJ, Kimble R. Unrecognized medical emergencies admitted to psychiatric units. Am J Emerg Med. 2000 Jul;18(4):390-3. PubMed PMID:10919525.

7. Haute Autorité de Santé - Guide - Affection Longue Durée - Affections psychiatriques de longue durée Troubles dépressifs récurrents ou persistants de l'adulte. Février 2009 Disponible https://www.hassante.fr/portail/upload/docs/application/pdf/2009-04/gm_ald23_troubles_depressifs_webavril2009.pdf

8. Fédération Française de Psychiatrie. Recommandation de bonne pratique en psychiatrie : Comment améliorer la prise en charge somatique des patients ayant une pathologie psychiatrique sévère et chronique. Recommandations Juin $2015 \quad$ Disponible : http://www.psydocfrance.fr/conf\%26rm/rpc/Reco_Soins_Soma_Psy.pdf

9. Nierenberg AA, Amsterdam JD. Treatment-resistant depression: definition and treatment approaches. J Clin Psychiatry. 1990 Jun;51 Suppl:39-47; discussion 48-50. Review. PubMed PMID: 2112132.

10. Decaux G (2006) Is asymptomatic hyponatremia really asymptomatic? Am J Med 119(7 Suppl. 1):S79-S82

11. Felger JC, Haroon E, Patel TA, Goldsmith DR, Wommack EC, Woolwine BJ, Le NA, Feinberg R, Tansey MG, Miller AH. What does plasma CRP tell us about peripheral and central inflammation in depression? Mol Psychiatry. 2018 Jun 12. doi:10.1038/s41380-018-0096-3.

12. Gilbody S, Lightfoot T, Sheldon T. Is low folate a risk factor for depression? A meta-analysis and exploration of heterogeneity. J Epidemiol Community Health. 2007 Jul;61(7):631-7. Review. PubMed PMID: 17568057;

13. Huang X, Fan Y, Han X, Huang Z, Yu M, Zhang Y, Xu Q, Li X, Wang X, Lu C, Xia Y. Association between Serum Vitamin Levels and Depression in U.S. Adults 20 Years or Older Based on National Health and Nutrition Examination Survey 2005-2006. Int J Environ Res Public Health. 2018 Jun 9;15(6). pii: E1215. doi:10.3390/ijerph15061215

14. Issac TG, Soundarya S, Christopher R, Chandra SR. Vitamin B12 deficiency: an important reversible comorbidity in neuropsychiatric manifestations. Indian J Psychol Med. 2015 Jan-Mar;37(1):26-9. doi: 10.4103/0253-7176.150809

15. Mouchabac S. Homocystéine, hyperhomocystéinémie et dépression .Neuropsychiatrie : Tendances et Débats $2008 ; 32: 9$ - 18 
16. Cohen BM, Sommer BR, Vuckovic A. Antidepressant-Resistant Depression in Patients With Comorbid Subclinical Hypothyroidism or High-Normal TSH Levels. Am J Psychiatry. 2018 Jul 1;175(7):598-604. doi: 10.1176/appi.ajp.2017.17080949

17. Schoretsanitis G, Paulzen M, Unterecker S, Schwarz M, Conca A, Zernig G, Gründer G, Haen E, Baumann P, Bergemann N, Clement HW, Domschke K, Eckermann G, Egberts K, Gerlach M, Greiner C, Havemann-Reinecke U, Hefner G, Helmer R, Janssen G, Jaquenoud-Sirot E, Laux G, Messer T, Mössner R, Müller MJ, Pfuhlmann B, Riederer P, Saria A, Schoppek B, Silva Gracia M, Stegmann B, Steimer W, StinglJC, Uhr M, Ulrich S, Waschgler R, Zurek G, Hiemke C. TDM in psychiatry and neurology: A comprehensive summary of the consensus guidelines for therapeutic drug monitoring in neuropsychopharmacology, update 2017; a tool for clinicians<sup/>. World J Biol Psychiatry. 2018 Apr;19(3):162-174. doi:10.1080/15622975.2018.1439595

18. Quaranta S, Dupouey J, Colle R, Verstuyft C. Pharmacogenetics of antidepressant drugs: State of the art and clinical implementation - recommendations from the French National Network of Pharmacogenetics. Therapie. 2017 Apr;72(2):311-318. doi: 10.1016/j.therap.2016.09.018

19. Tessier C, Sweers K, Frajerman A, Bergaoui H, Ferreri F, Delva C, Lapidus N, Lamaziere A, Roiser JP, De Hert $M$, Nuss $P$. Membrane lipidomics in schizophrenia patients: a correlational study with clinical and cognitive manifestations. Transl Psychiatry. 2016 Oct 4;6(10):e906. doi: 10.1038/tp.2016.142

20. Kasote DM, Hegde MV, Katyare SS. Mitochondrial dysfunction in psychiatric and neurological diseases: cause(s), consequence(s), and implications of antioxidant therapy. Biofactors. 2013 Jul-Aug;39(4):392-406. doi: 10.1002/biof.1093

21. Ferrafiat V, Raffin M, Freri E, Granata T, Nardocci N, Zibordi F, Bodeau N, Benarous X, Olliac B, Riquin E, Xavier J, Viaux S, Haroche J, Amoura Z, Gerardin P, Cohen D, Consoli A. A causality algorithm to guide diagnosis and treatment of catatonia due to autoimmune conditions in children and adolescents. Schizophr Res. 2017 Jun 25. pii: S0920-9964(17)30375-4. doi: 10.1016/j.schres.2017.06.036

22. Bonnot O, Klünemann HH, Sedel F, Tordjman S, Cohen D, Walterfang M. Diagnostic and treatment implications of psychosis secondary to treatable metabolic disorders in adults: a systematic review. Orphanet J Rare Dis. 2014 Apr 28;9:65. doi: 10.1186/1750-1172-9-65. Review.

23. Warren N, Siskind D, O'Gorman C. Refining the psychiatric syndrome of anti-N-methyl-d-aspartate receptor encephalitis. Acta Psychiatr Scand. 2018 Jul 10. doi: 10.1111/acps.12941

24. Kimberlin DW. Management of HSV encephalitis in adults and neonates: diagnosis, prognosis and treatment. Herpes. 2007 Jun;14(1):11-6. Review

25. Noblett J, Roberts E. The importance of not jumping to conclusions: syphilis as an organic cause of neurological, psychiatric and endocrine presentations. BMJ Case Rep. 2015 Feb 25;2015. pii: bcr2014207900. doi: 10.1136/bcr-2014-207900.

26. Seo EH, Yang HJ, Kim SH, Park JH, Yoon HJ. Psychotic mania as the solitary manifestation of neurosyphilis. Ann Gen Psychiatry. 2018 Jun 6;17:24. doi:10.1186/s12991-018-0195-1. eCollection 2018

27. Alciati A, Fusi A, D'Arminio Monforte A, Coen M, Ferri A, Mellado C. New-onset delusions and hallucinations in patients infected with HIV. J Psychiatry Neurosci. 2001 May;26(3):229-34. PubMed PMID: 11394192

28. Fallon BA, Nields JA, Parsons B, Liebowitz MR, Klein DF. Psychiatric manifestations of Lyme borreliosis. J Clin Psychiatry. 1993 Jul;54(7):263-8. PubMed PMID: 8335653.

29. Blanc F, Ben Abdelghani K, Schramm F, Jaulhac B, Chatelus E, Sordet C, Gottenberg JE, Sibilia J. Whipple limbic encephalitis. Arch Neurol. 2011 Nov;68(11):1471-3. doi: 10.1001/archneurol.2011.532

30. Graus F, Delattre JY, Antoine JC, et al. Recommended diagnostic criteria for paraneoplastic neurological syndromes. JNNP 2004;75:1135-40 
31. Venkatesan A, Tunkel AR, Bloch KC, Lauring AS, Sejvar J, Bitnun A, et al. . Case definitions, diagnostic algorithms, and priorities in encephalitis: consensus statement of the international encephalitis consortium. Clin Infect Dis. (2013) 57:1114-28. 10.1093/cid/cit458

32. Lefèvre G, Zéphir H, Michelin E, Semah F, Warembourg F, Pruvo JP, Hachulla E, Lenfant P, Dubucquoi S, Vermersch P, Hatron PY, Prin L, Launay D. [Neuropsychiatric systemic lupus erythematosus (2nd part). Diagnostic and treatment tools in psychiatric or central nervous system manifestations in systemic lupus erythematosus]. Rev Med Interne. 2012 Sep;33(9):503-13. doi: 10.1016/j.revmed.2012.03.354. Epub 2012 May 9. Review

33. Zhou JY, Xu B, Lopes J, Blamoun J, Li L. Hashimoto encephalopathy: literature review. Acta Neurol Scand. 2017 Mar;135(3):285-290. doi: 10.1111/ane.12618. Epub 2016 Jun 20. Review

34. Marrie RA, Reingold S, Cohen J, Stuve O, Trojano M, Sorensen PS, Cutter G, Reider N. The incidence and prevalence of psychiatric disorders in multiple sclerosis: a systematic review. Mult Scler. 2015 Mar;21(3):30517. doi: 10.1177/1352458514564487. Epub 2015 Jan 12. Review

35. Jamison NK, Holmes EG. A Case of Mast Cell Leukemia: A Review of the Pathophysiology of Systemic Mastocytosis and Associated Psychiatric Symptoms. Psychosomatics. 2018 Feb 12. pii: S0033-3182(18)30129-4. doi: 10.1016/j.psym.2018.02.002

36. Lyketsos CG, Carrillo MC, Ryan JM, Khachaturian AS, Trzepacz P, Amatniek J, Cedarbaum J, Brashear R, Miller DS. Neuropsychiatric symptoms in Alzheimer's disease. Alzheimers Dement. 2011 Sep;7(5):532-9. doi: 10.1016/j.jalz.2011.05.2410.

37. Weintraub D, Stern MB. Psychiatric complications in Parkinson disease. Am J Geriatr Psychiatry. 2005 Oct;13(10):844-51. Review

38. Ballard CG, Jacoby R, Del Ser T, Khan MN, Munoz DG, Holmes C, Nagy Z, Perry EK, Joachim C, Jaros E, O'Brien JT, Perry RH, McKeith IG. Neuropathological substrates of psychiatric symptoms in prospectively studied patients with autopsy-confirmed dementia with lewy bodies. Am J Psychiatry. 2004 May;161(5):843-9.

39. Dols A, van Liempt S, Gossink F, Krudop WA, Sikkes S, Pijnenburg YA, Stek ML. Identifying Specific Clinical Symptoms of Behavioral Variant Frontotemporal Dementia Versus Differential Psychiatric Disorders in Patients Presenting With a Late-Onset Frontal Lobe Syndrome. J Clin Psychiatry. 2016 Oct;77(10):1391-1395. doi: 10.4088/JCP.15m09844

40. Gold JA, Sher Y, Maldonado JR. Frontal Lobe Epilepsy: A Primer for Psychiatrists and a Systematic Review of Psychiatric Manifestations. Psychosomatics. 2016 Sep-Oct;57(5):445-64. doi: 10.1016/j.psym.2016.05.005

41. Kandratavicius L, Lopes-Aguiar C, Bueno-Júnior LS, Romcy-Pereira RN, Hallak JE, Leite JP. Psychiatric comorbidities in temporal lobe epilepsy: possible relationships between psychotic disorders and involvement of limbic circuits. Rev Bras Psiquiatr. 2012 Dec;34(4):454-66. Review

42. Madhusoodanan S, Ting MB, Farah T, Ugur U. World J Psychiatry. 2015 Sep 22;5(3):273-85. Psychiatric aspects of brain tumors: A review.

43. Anglin RE, Rosebush PI, Mazurek MF. The neuropsychiatric profile of Addison's disease: revisiting a forgotten phenomenon. J Neuropsychiatry Clin Neurosci. 2006 Fall;18(4):450-9. Review

44. Alguire C, Chbat J, Forest I, Godbout A, Bourdeau I. Unusual presentation of pheochromocytoma: thirteen years of anxiety requiring psychiatric treatment. Endocrinol Diabetes Metab Case Rep. 2018 Apr 6;2018. pii: 170176. doi:10.1530/EDM-17-0176. eCollection 2018

45. Ramaekers V, Sequeira JM, Quadros EV. Clinical recognition and aspects of the cerebral folate deficiency syndromes. Clin Chem Lab Med. 2013 Mar 1;51(3):497-511. doi: 10.1515/cclm-2012-0543 
46. Ramaekers VT, Sequeira JM, Quadros EV. The basis for folinic acid treatment in neuro-psychiatric disorders. Biochimie. 2016 Jul;126:79-90. doi:10.1016/j.biochi.2016.04.005. Epub 2016 Apr 8. Review

47. Mukku SSR, Suhas S, Thippeswamy H, Ganjekar S, Arvind HR, Chaturvedi SK. Mixed neuropsychiatric clinical manifestations associated with vitamin B12 deficiency. Asian J Psychiatr. 2018 May 23;36:25-27. doi: 10.1016/j.ajp.2018.05.026

48. Maubert A, Hanon C, Sedel F. [Psychiatric disorders in adult form of Niemann-Pick disease type C]. Encephale. 2016 Jun;42(3):208-13. doi:10.1016/j.encep.2015.11.003

49. Fraidakis MJ. Psychiatric manifestations in cerebrotendinous xanthomatosis. Transl Psychiatry. 2013 Sep 3;3:e302. doi: 10.1038/tp.2013.76.

50. Zimbrean P, Seniów J. Cognitive and psychiatric symptoms in Wilson disease. Handb Clin Neurol. 2017;142:121-140. doi: 10.1016/B978-0-444-63625-6.00011-2

51. Serata D, Del Casale A, Rapinesi C, Mancinelli I, Pompili P, Kotzalidis GD, Aimati L, Savoja V, Sani G, Simmaco M, Tatarelli R, Girardi P. Hemochromatosis-induced bipolar disorder: a case report. Gen Hosp Psychiatry. 2012 Jan-Feb;34(1):101.e1-3. doi: 10.1016/j.genhosppsych.2011.04.013

52. Duque-Serrano L, Patarroyo-Rodriguez L, Gotlib D, Molano-Eslava JC. Psychiatric Aspects of Acute Porphyria: a Comprehensive Review Current Psychiatry Reports (2018) 20: 5

53. MacQueen GM, Rosebush PI, Mazurek MF. Neuropsychiatric aspects of the adult variant of Tay-Sachs disease. J Neuropsychiatry Clin Neurosci. 1998 Winter;10(1):10-9. Review

54. Kölker S, Garcia-Cazorla A, Valayannopoulos V, Lund AM, Burlina AB, Sykut-Cegielska J, Wijburg FA, Teles EL, Zeman J, Dionisi-Vici C, Barić I, Karall D, Augoustides-Savvopoulou P, Aksglaede L, Arnoux JB, Avram P, Baumgartner MR, Blasco-Alonso J, Chabrol B, Chakrapani A, Chapman K, I Saladelafont EC, Couce ML, de Meirleir L, Dobbelaere D, Dvorakova V, Furlan F, Gleich F, Gradowska W, Grünewald S, Jalan A, Häberle J, Haege G, Lachmann R, Laemmle A, Langereis E, de Lonlay P, Martinelli D, Matsumoto S, Mühlhausen C, de Baulny HO, Ortez C,Peña-Quintana L, Ramadža DP, Rodrigues E, Scholl-Bürgi S, Sokal E, Staufner C, Summar ML, Thompson N, Vara R, Pinera IV, Walter JH, Williams M, Burgard P. The phenotypic spectrum of organic acidurias and urea cycle disorders. Part 1: the initial presentation. J Inherit Metab Dis. 2015 Nov;38(6):1041-57. doi:10.1007/s10545-015-9839-3. Epub 2015 Apr 15. Erratum in: J Inherit Metab Dis.2015 Nov;38(6):1155-6. Cazorla, Angeles Garcia [corrected to Garcia-Cazorla, Angeles

55. Sacharow SJ, Picker JD, Levy HL. Homocystinuria Caused by Cystathionine Beta-Synthase Deficiency. 2004 Jan 15 [updated 2017 May 18]. In: Adam MP, Ardinger HH, Pagon RA, Wallace SE, Bean LH, Stephens K, Amemiya A, editors. GeneReviews ${ }^{\circledR}$ [Internet]. Seattle (WA): University of Washington, Seattle; $1993-2018$. Available from http://www.ncbi.nlm.nih.gov/books/NBK1524

56. Gales A, Masingue M, Millecamps S et al. Adolescence/adult onset MTHFR deficiency may manifest as isolated and treatable distinct neuro-psychiatric syndromes. Orphanet Journal of Rare Diseases (2018) 13:29

57. Bilder DA, Noel JK, Baker ER, Irish W, Chen Y, Merilainen MJ, Prasad S, Winslow BJ. Systematic Review and Meta-Analysis of Neuropsychiatric Symptoms and Executive Functioning in Adults With Phenylketonuria. Dev Neuropsychol. 2016 May-Jun;41(4):245-260.

58. Bar-Yam, Yaneer (2002). "General Features of Complex Systems" (PDF). Encyclopedia of Life Support Systems. EOLSS UNESCO Publishers, Oxford, UK. Retrieved 16 September 2014

59. Kraemer HC, Stice E, Kazdin A, Offord D, Kupfer D. How do risk factors work together? Mediators, moderators, and independent, overlapping, and proxy risk factors. Am J Psychiatry. 2001 Jun;158(6):848-56. Review. 
60. Caisse Nationale d’Assurance Maladie - Améliorer la qualité du système de santé et maîtriser les dépenses Rapport au ministre chargé de la Sécurité sociale et au Parlement sur l'évolution des charges et produits de l'Assurance Maladie au titre de 2019 - https://www.ameli.fr/l-assurance-maladie/statistiques-etpublications/rapports-et-periodiques/rapports-charges-produits-de-l-assurance-maladie/rapports-charges-etproduits-pour-2018-et-2019/rapport-charges-et-produits-pour-l-annee-2019.php 